\title{
First-line chemotherapy with capecitabine/oxaliplatin for advanced gastric cancer: A phase I study
}

\author{
HIRONAGA SATAKE $^{1}$, HISATERU YASUI ${ }^{1}$, TAKESHI KOTAKE ${ }^{1}$, YOSHIHIRO OKITA $^{2}$, \\ YUKIMASA HATACHI ${ }^{1}$, MASAHITO KOTAKA ${ }^{3}$, TAKESHI KATO ${ }^{4}$ and AKIHITO TSUJI ${ }^{2}$
}

\author{
${ }^{1}$ Department of Medical Oncology, Kobe City Medical Center General Hospital, Kobe, Hyogo 650-0047; ${ }^{2}$ Department \\ of Clinical Oncology, Kagawa University Hospital, Kita, Kagawa 761-0793; ${ }^{3}$ Gastrointestinal Cancer Center, Sano Hospital, \\ Kobe, Hyogo 655-0031; ${ }^{4}$ Department of Surgery, Kansai Rosai Hospital, Amagasaki, Hyogo 660-8511, Japan
}

Received February 24, 2017; Accepted May 22, 2017

DOI: $10.3892 / \mathrm{mco} .2017 .1335$

\begin{abstract}
Combination chemotherapy with capecitabine and oxaliplatin for gastric cancer (G-XELOX) is considered as a potentially promising regimen. However, the use of the G-XELOX regimen in Japanese patients has not been investigated to date, and recommended doses of G-XELOX for Japanese patients with metastatic gastric cancer have not been established. The aim of the present study was to determine the maximum tolerated dose (MTD) and recommended dose (RD) for systemic chemotherapy with G-XELOX for metastatic gastric cancer. The enrolled patients received systemic chemotherapy with oxaliplatin $130 \mathrm{mg} / \mathrm{m}^{2}$ on day 1 and capecitabine $2,000 \mathrm{mg} / \mathrm{m}^{2} /$ day, b.i.d. for 14 days, repeated every 3 weeks. A decrease in oxaliplatin dose was planned from start level 1 $\left(130 \mathrm{mg} / \mathrm{m}^{2}\right)$. A total of 6 patients were enrolled between January and July 2015. MTD was not reached at level 1. Oxaliplatin $130 \mathrm{mg} / \mathrm{m}^{2}$ in combination with capecitabine $2,000 \mathrm{mg} / \mathrm{m}^{2} /$ day b.i.d. could be administered with acceptable toxicity, and
\end{abstract}

Correspondence to: Dr Hironaga Satake, Department of Medical Oncology, Kobe City Medical Center General Hospital, 2-1-1 Minatojima Minamimachi, Chuo-ku, Kobe, Hyogo 650-0047, Japan

E-mail: takeh1977@gmail.com

Abbreviations: G-XELOX, combination chemotherapy with capecitabine and oxaliplatin for advanced gastric cancer; MTD, maximum tolerated dose; RD, recommended dose; b.i.d., twice per day; UMIN, university hospital medical network; RECIST, Response Evaluation Criteria In Solid Tumors; AGC, advanced or metastatic gastric cancer; ECF, epirubicin, cisplatin and 5-fluorouracil; ECOG, Eastern Cooperative Oncology Group; Hb, hemoglobin; ANC, absolute neutrophil count; DLT, dose-limiting toxicity; CT, computed tomography; PFS, progression-free survival; OS, overall survival; RR, response rate; DCR, disease control rate; SOX, S-1 and oxaliplatin; CS, cisplatin plus S-1; REAL-2, randomized ECF for advanced and locally advanced esophagogastric cancer 2

Key words: gastric cancer, systemic chemotherapy, capecitabine, oxaliplatin, XELOX, CapeOx, phase I study all patients were treated at these doses. One case of grade 3 stomatitis was considered as a dose-limiting toxicity at level 1; however, excluding this case, no grade 3 or 4 non-hematological toxicity was observed. There were no treatment-related deaths. The median relative dose intensity was $71.3 \%$ for capecitabine and $92.1 \%$ for oxaliplatin. Of the 6 patients, 3 had measurable lesions according to the Response Evaluation Criteria In Solid Tumors; the response rate and disease control rate were both $67 \%$. Therefore, systemic chemotherapy with G-XELOX was well-tolerated by patients with advanced gastric cancer. The $\mathrm{RD}$ was defined as oxaliplatin $130 \mathrm{mg} / \mathrm{m}^{2}$ in combination with capecitabine $2,000 \mathrm{mg} / \mathrm{m}^{2} /$ day b.i.d.

\section{Introduction}

Gastric cancer is the fifth most frequently diagnosed malignancy and the third leading cause of cancer-related mortality worldwide (1). Once the disease becomes inoperable, the prognosis for gastric cancer is exceptionally poor. The majority of the cases of inoperable advanced or metastatic gastric cancer (AGC) remain incurable, with a median survival of only 11-14 months, even for patients who undergo chemotherapy (2-4). The standard treatment for AGC currently consists of systemic chemotherapy; however, despite several randomized trials, a consensus standard chemotherapy regimen for AGC has not been established. The combination of fluoropyrimidine and platinum is used worldwide for the treatment of AGC (2,3), and cisplatin plus 5-fluorouracil (FU) or epirubicin plus cisplatin plus 5-FU (ECF) are widely used (5). However, the administration of cisplatin is limited by nephrotoxicity, which is the dose-limiting toxicity of this agent. In Japan, in order to reduce nephrotoxicity, a 24-h hydration period is recommended following administration of cisplatin.

Oxaliplatin, a non-nephrotoxic platinum analog, is reported to be as effective as cisplatin, with a favorable safety profile in patients with AGC $(2,4)$. Furthermore, the oral fluoropyrimidines capecitabine and S-1 have been developed as substitutes for 5-FU, which is administered by continuous infusion via a central venous catheter. Capecitabine (Xeloda, Roche) has been shown to be effective in the treatment of gastric cancer, and is also administered as a combination treatment with 
oxaliplatin (6). Capecitabine plus oxaliplatin (G-XELOX) is considered as a standard regimen for AGC. Globally, doses in the G-XELOX regimen consist of capecitabine $\left(1,000 \mathrm{mg} / \mathrm{m}^{2}\right.$ twicedailyondays $\left.1-14\right)$ plusoxaliplatin $\left(130 \mathrm{mg} / \mathrm{m}^{2}\right.$ on day 1 ) every 3 weeks.

However, the use of the G-XELOX regimen in Japanese patients has not been described to date, and recommended doses for Japanese patients with AGC have not been established.

The aim of the present study was to determine the maximum tolerated dose (MTD) and recommended dose (RD) for chemotherapy with oxaliplatin combined with a fixed capecitabine dose in the treatment of AGC.

\section{Patients and methods}

Eligibility criteria and patients. The eligibility criteria for inclusion in the present study were as follows: Age $\geq 20$ years; histologically confirmed human epidermal growth factor receptor type 2-negative unresectable or recurrent gastric cancer; Eastern Cooperative Oncology Group (ECOG) performance status $<2$; estimated life expectancy $\geq 3$ months; and adequate organ function, as defined by hemoglobin $(\mathrm{Hb})$ $\geq 8 \mathrm{~g} / \mathrm{dl}$, absolute neutrophil count (ANC) $\geq 1,500 / \mathrm{mm}^{3}$, platelet count $\geq 100,000 / \mathrm{mm}^{3}$, total bilirubin $\leq 1.5 \mathrm{mg} / \mathrm{dl}$, serum transaminase level $\leq 100 \mathrm{U} / \mathrm{l}$ and creatinine clearance $\geq 40 \mathrm{ml} / \mathrm{min}$. The exclusion criteria were as follows: Contraindication to either drug included in the chemotherapy regimen; evidence of prior history of platinum administration; insufficient oral intake; synchronous or previous malignancy other than carcinoma in situ; or severe comorbidities.

This trial was conducted in accordance with the Helsinki Declaration and Good Clinical Practice guidelines, and was approved by the Institutional Review Board of Kobe City Medical Center General Hospital (Kobe, Japan). All the patients were required to provide written informed consent prior to enrolment.

Study design and treatment. Protocol treatment was defined as chemotherapy consisting of capecitabine and oxaliplatin. The patients received capecitabine $1,000 \mathrm{mg} / \mathrm{m}^{2}$ b.i.d. on days 1-14 plus oxaliplatin $130 \mathrm{mg} / \mathrm{m}^{2}$ every 3 weeks. The study was designed to determine the recommended dose (RD) of chemotherapy. A total of 6 patients were treated at dose level 1 (capecitabine $1,000 \mathrm{mg} / \mathrm{m}^{2}$ b.i.d. on days $1-14$ and oxaliplatin $130 \mathrm{mg} / \mathrm{m}^{2}$ on day 1). If $\geq 3$ of the 6 patients experienced a dose-limiting toxicity (DLT), an additional 6 patients were accrued at the next lower dose level (level 0; capecitabine $1,000 \mathrm{mg} / \mathrm{m}^{2}$ twice daily on days $1-14$ and oxaliplatin $100 \mathrm{mg} / \mathrm{m}^{2}$ on day 1). The MTD was defined as the dose at which $\geq 3$ of the 6 patients experienced a DLT. Treatment was repeated until disease progression, unacceptable toxicity, or withdrawal of consent. Treatment was delayed if, on the planned day of treatment, the laboratory results included any of the following: ANC $<1,500 / \mathrm{mm}^{3}$, platelet count $<75,000 / \mathrm{mm}^{3}, \mathrm{Hb}<8 \mathrm{~g} / \mathrm{dl}$, serum transaminase $>100 \mathrm{U} / 1$, total bilirubin $>2.0 \mathrm{mg} / \mathrm{dl}$, or serum creatinine $>1.50 \mathrm{mg} / \mathrm{dl}$, or if symptomatic toxicity was present. Patients who could not tolerate oxaliplatin continued to receive capecitabine monotherapy until disease progression or intolerable toxicity. The RD was defined as one dose level below the MTD. If the MTD was not achieved, even at level 1 , it was considered as the RD. DLT was defined as any of the following adverse events occurring in the first cycle: i) Grade 4 neutropenia lasting $>4$ days; ii) grade 4 thrombocytopenia $\left(<25,000 / \mathrm{mm}^{3}\right)$; iii) febrile neutropenia; iv) grade 3 or 4 non-hematological toxicities; v) treatment discontinuation due to adverse events; or vi) treatment-related death. Protocol treatment was administered triweekly until disease progression, unacceptable toxicity, or withdrawal of consent. In patients with pharyngolaryngeal dysesthesia, the duration of oxaliplatin infusion was prolonged from 2-6 h. In the event of grade 4 non-hematological toxicities, treatment was definitively interrupted.

Study assessment. Pretreatment evaluation included a medical history, physical examination, complete blood cell count and serum chemistry tests, esophagogastroduodenoscopy, and chest, abdominal and pelvic computed tomography (CT) scans. Clinical examination and biochemical tests were required before and during each treatment cycle. All images for tumor responses were evaluated according to the Response Evaluation Criteria In Solid Tumors (RECIST), version 1.1 (7). All adverse events during chemotherapy were evaluated using the National Cancer Institute Common Terminology Criteria for Adverse Events, version 4.0 (https://www. eortc.be/services/doc/ctc/CTCAE_4.03_2010-06-14_Quick Reference_5x7.pdf).

Endpoints and statistical analysis. The primary endpoint in the present study was the MTD and RD of the G-XELOX regimen. Secondary endpoints included toxicities, response rate (RR), progression-free survival (PFS) and overall survival (OS). Safety and efficacy analyses were both conducted in an intention-to-treat population, defined as all patients enrolled in the study who received at least one dose of chemotherapy. All statistical analyses were conducted using the SPSS software package (SPSS 22.0 Inc., IBM Corp., Armonk, NY, USA).

This trial was registered with the University Hospital Medical Information Network (UMIN no 000015951).

\section{Results}

Patients. Between January and July 2015, 6 patients were enrolled. The characteristics of the enrolled patients are listed in Table I. The median age was 72 years, $67 \%$ of the patients had diffuse-type disease, $50 \%$ had multiple organ metastases, and all patients were chemo-naïve. One patient had undergone distal gastrectomy for resection of the primary tumor.

Treatment administration and DLT. A total of 6 patients were enrolled at dose level 1 (capecitabine $1,000 \mathrm{mg} / \mathrm{m}^{2}$ b.i.d. on days $1-14$ and oxaliplatin $130 \mathrm{mg} / \mathrm{m}^{2}$ on day 1). Of the 6 patients administered level 1,1 patient developed a DLT (grade 3 stomatitis) and, hence, the RD for phase II studies was determined to be capecitabine $1,000 \mathrm{mg} / \mathrm{m}^{2}$ b.i.d. on days $1-14$ and oxaliplatin $130 \mathrm{mg} / \mathrm{m}^{2}$ on day 1 .

Toxicity and dose intensity. Toxicity was assessable in all 6 patients. The most severe toxicities throughout the protocol treatment period are listed in Table II. Grade $\geq 3$ 
Table I. Patient characteristics $(n=6)$.

\begin{tabular}{|c|c|c|}
\hline Variables & $\mathrm{n}$ & $\%$ \\
\hline \multicolumn{3}{|l|}{ Age, years } \\
\hline Median & 72 & \\
\hline Range & $65-77$ & \\
\hline \multicolumn{3}{|l|}{ Sex } \\
\hline Male & 3 & 50 \\
\hline Female & 3 & 50 \\
\hline \multicolumn{3}{|l|}{ ECOG PS } \\
\hline 0 & 3 & 50 \\
\hline 1 & 3 & 50 \\
\hline \multicolumn{3}{|c|}{ Primary tumor location } \\
\hline Upper & 3 & 50 \\
\hline Middle & 3 & 50 \\
\hline Lower & 0 & 0 \\
\hline \multicolumn{3}{|l|}{ Histology } \\
\hline Intestinal & 2 & 33 \\
\hline Diffuse & 4 & 67 \\
\hline \multicolumn{3}{|c|}{ Surgery for primary tumor } \\
\hline Yes & 1 & 17 \\
\hline No & 5 & 83 \\
\hline \multicolumn{3}{|c|}{ Prior adjuvant chemotherapy } \\
\hline Yes & 0 & 0 \\
\hline No & 6 & 100 \\
\hline \multicolumn{3}{|l|}{ Metastatic site } \\
\hline Single & 3 & 50 \\
\hline Multiple & 3 & 50 \\
\hline \multicolumn{3}{|c|}{ Peritoneal metastasis } \\
\hline Yes & 1 & 17 \\
\hline No & 5 & 83 \\
\hline \multicolumn{3}{|l|}{ HER2 status } \\
\hline IHC 0 & 3 & 50 \\
\hline IHC 1 & 2 & 33 \\
\hline $\mathrm{IHC}^{2 / \mathrm{FISH}^{-}}$ & 1 & 17 \\
\hline
\end{tabular}

ECOG, Eastern Cooperative Oncology Group; PS, performance status; HER2, human epidermal growth factor receptor type 2; IHC, immunohistochemistry; FISH, fluorescence in situ hybridization.

thrombocytopenia and febrile neutropenia occurred in 17 and $0 \%$ of the patients, respectively. Grade $\geq 3$ non-hematological toxicity (stomatitis) only occurred in 1 patient (17\%), but it subsided with conservative treatment. Peripheral neuropathy was observed in all patients, but without functional disorders. The median percentage of relative dose intensity delivered during protocol treatment was $71.3 \%$ (range, 12.4-100\%) for capecitabine and $92.1 \%$ (range, $68.3-100 \%$ ) for oxaliplatin.

Efficacy and treatment continuation. Response was assessable in 5 patients. Of the 6 patients, 3 had measurable lesions according to RECIST; of those 3 patients, 2 had a partial response and 1 had progressive disease, with an RR of $67 \%$
Table II. Maximum toxicity per patient during protocol treatment $(n=6)$.

\begin{tabular}{|c|c|c|c|c|c|c|}
\hline \multirow{2}{*}{$\begin{array}{l}\text { Adverse events } \\
\text { Hematological }\end{array}$} & \multicolumn{6}{|c|}{ NCI-CTC grade } \\
\hline & 1 & 2 & 3 & 4 & All, \% & $3 / 4, \%$ \\
\hline Leukopenia & 3 & 0 & 0 & 0 & 50 & 0 \\
\hline Neutropenia & 2 & 1 & 0 & 0 & 50 & 0 \\
\hline Anemia & 6 & 0 & 0 & 0 & 100 & 0 \\
\hline Thrombocytopenia & 4 & 0 & 1 & 0 & 83 & 17 \\
\hline \multicolumn{7}{|l|}{ Non-hematological } \\
\hline Anorexia & 3 & 2 & 0 & 0 & 83 & 0 \\
\hline Ascites & 1 & 0 & 0 & 0 & 17 & 0 \\
\hline Cheilitis & 0 & 1 & 0 & 0 & 17 & 0 \\
\hline Conjunctivitis & 0 & 1 & 0 & 0 & 17 & 0 \\
\hline Constipation & 1 & 0 & 0 & 0 & 17 & 0 \\
\hline Diarrhea & 2 & 1 & 0 & 0 & 50 & 0 \\
\hline Dizziness & 1 & 0 & 0 & 0 & 17 & 0 \\
\hline Edema & 1 & 0 & 0 & 0 & 17 & 0 \\
\hline Fatigue & 5 & 1 & 0 & 0 & 100 & 0 \\
\hline Febrile neutropenia & - & - & 0 & 0 & 0 & 0 \\
\hline Hand-foot syndrome & 1 & 0 & 0 & 0 & 17 & 0 \\
\hline Nausea/vomiting & 3 & 1 & 0 & 0 & 67 & 0 \\
\hline Neurotoxicity & 5 & 1 & 0 & 0 & 100 & 0 \\
\hline Pain & 2 & 0 & 0 & 0 & 33 & 0 \\
\hline Stomatitis & 0 & 0 & 1 & 0 & 17 & 17 \\
\hline
\end{tabular}

NCI-CTC, national cancer institute common toxicity criteria.

and a disease control rate (DCR) of $67 \%$. The median time to the first dose reduction was 2 cycles (range, 2-3 cycles) in 4 of the 6 patients, commonly due to gastrointestinal toxicities or myelosuppression. Of the 6 patients, 4 discontinued the protocol treatment due to disease progression and 1 due to toxicities. One patient underwent curative resection for primary disease after 11 cycles of protocol treatment and remained alive without disease at the time of writing, $>2$ years after the initiation of the protocol treatment (last follow-up, April 2017). The median PFS and OS in all the patients were 3.6 and 5.7 months, respectively.

\section{Discussion}

To the best of our knowledge, this is the first report of the feasibility and activity of systemic chemotherapy consisting of capecitabine plus oxaliplatin (G-XELOX) in Japanese patients with metastatic gastric cancer. RDs of systemic chemotherapy with capecitabine plus oxaliplatin were defined as oxaliplatin at $130 \mathrm{mg} / \mathrm{m}^{2}$ in combination with capecitabine at $2,000 \mathrm{mg} / \mathrm{m}^{2} /$ day b.i.d.

Allowing for the small number of patients in this study, the safety of G-XELOX appeared to be promising. The most frequent adverse events were anemia, fatigue and peripheral neurotoxicity. In addition, all 6 patients developed grade 1 anemia. Several patients with advanced gastric cancer experience some degree of anemia, and all 6 patients in this study had the equivalent of grade 1 anemia (<lower limit of 
normal, $10.0 \mathrm{~g} / \mathrm{dl}$ ) at enrollment (median, $12.0 \mathrm{~g} / \mathrm{dl}$ ). Grade $\geq 3$ toxicities (thrombocytopenia and stomatitis) occurred in $17 \%$ of the cases $(n=2)$, but both cases resolved. In this study of G-XELOX, all-grade thrombocytopenia occurred in $83 \%$ of the cases, and grade 3 thrombocytopenia occurred after 3 cycles of chemotherapy. A phase III study comparing oxaliplatin plus S-1 with cisplatin plus S-1 (CS) as first-line chemotherapy for AGC, reported that thrombocytopenia at any grade was more frequently observed in the oxaliplatin group (4). Thrombocytopenia is considered to be a characteristic toxicity of oxaliplatin-based regimens compared with cisplatin-based regimens.

A recent phase III study of epirubicin/fluoropyrimidine/platinum triplet (REAL-2) and a phase III study comparing SOX with CS, indicated that oxaliplatin is superior to cisplatin in terms of efficacy as well as tolerability $(2,4)$. The G-XELOX regimen requires only one clinical visit per 3-week cycle for a 2 -h infusion of oxaliplatin, conferring a marked advantage regarding disruption of daily life over regimens containing cisplatin, which require hospitalization to ensure hydration.

Although efficacy was not the primary endpoint of the present study, antitumor activity (RR=67\% and $\mathrm{DCR}=67 \%$ ) was highly promising. A phase II study of G-XELOX achieved an overall RR of 63\%, a median PFS of 5.8 months, and a median OS of 11.9 months (8). Although only 3 of our 6 patients had measurable lesions according to RECIST, these results may confirm the efficacy of the G-XELOX regimen in the treatment of gastric cancer. The survival results were unsatisfactory, but may have been affected by the small study size and relatively advanced age of the enrolled patients. Furthermore, proton pump inhibitors (PPIs) may have impaired capecitabine efficacy. A recent analysis of the TRIO-013/LOGiC trial, a phase III randomized trial comparing capecitabine and oxaliplatin, with or without lapatinib, in metastatic gastroesophageal cancer, reported that PPIs negatively affect capecitabine efficacy by possibly raising gastric $\mathrm{pH}$ levels, leading to altered dissolution and absorption (9). In the present study, 5 of the 6 patients received PPIs and the remaining patient received a histamine receptor antagonist as gastric acid suppressant at the time of enrolment.

A limitation associated with the study design should also be discussed. Although a de-escalation design was initially planned for this study, the dose of oxaliplatin did not reach the MTD. Therefore, there remains the question whether oxaliplatin doses may be further increased in Japanese patients with advanced gastric cancer.

In conclusion, systemic chemotherapy with the G-XELOX regimen was found to be well-tolerated by patients with AGC. This phase I study demonstrated that the RDs of systemic chemotherapy with oxaliplatin and capecitabine was oxaliplatin $130 \mathrm{mg} / \mathrm{m}^{2}$ in combination with capecitabine $2,000 \mathrm{mg} / \mathrm{m}^{2} /$ day b.i.d.. This regimen demonstrated sufficient activity to warrant further phase II studies.

\section{Acknowledgements}

The authors would like to thank the patients and families who participated in this study. We also thank Rie Tamaki, Saori Tokuhara and Chiori Taniguchi for their support. Akihito Tsuji has received honoraria from Chugai Pharma, Takeda Pharmaceutical, Eli-Lilly, Taiho Pharma, Bayer, Bristol-Myers Squibb Japan, Daiichi Sankyo and Merck Serono. Masahito Kotaka has received honoraria from Chugai Pharma, Takeda Pharmaceutical, Yakult, Taiho Pharma and Merck Serono. Takeshi Kato has received honoraria and/or research funding from Chugai Pharma, Takeda Pharmaceutical, Eli-Lilly, Taiho Pharma, Bayer and Merck Serono.

\section{References}

1. Ferlay J, Soerjomataram I, Dikshit R, Eser S, Mathers C, Rebelo M, Parkin DM, Forman D and Bray F: Cancer incidence and mortality worldwide: Sources, methods and major patterns in GLOBOCAN 2012. Int J Cancer 136: E359-E386, 2015.

2. Cunningham D, Starling N, Rao S, Iveson T, Nicolson M, Coxon F, Middleton G, Daniel F, Oates J and Norman AR; Upper Gastrointestinal Clinical Studies Group of the National Cancer Research Institute of the United Kingdom: Capecitabine and oxaliplatin for advanced esophagogastric cancer. N Engl J Med 358: 36-46, 2008.

3. Koizumi W, Narahara H, Hara T, Takagane A, Akiya T, Takagi M, Miyashita K, Nishizaki T, Kobayashi O, Takiyama W, et al: S-1 plus cisplatin versus $\mathrm{S}-1$ alone for first-line treatment of advanced gastric cancer (SPIRITS trial): A phase III trial. Lancet Oncol 9: 215-221, 2008.

4. Yamada Y, Higuchi K, Nishikawa K, Gotoh M, Fuse N, Sugimoto N, Nishina T, Amagai K, Chin K, Niwa Y, et al: Phase III study comparing oxaliplatin plus S-1 with cisplatin plus S-1 in chemotherapy-naive patients with advanced gastric cancer. Ann Oncol 26: 141-148, 2015.

5. Rivera F, Vega-Villegas ME and López-Brea MF: Chemotherapy of advanced gastric cancer. Cancer Treat Rev 33: 315-324, 2007.

6. Kim GM, Jeung HC, Rha SY, Kim HS, Jung I, Nam BH, Lee KH and Chung HC: A randomized phase II trial of S-1-oxaliplatin versus capecitabine-oxaliplatin in advanced gastric cancer. Eur J Cancer 48: 518-526, 2012.

7. Eisenhauer EA, Therasse P, Bogaerts J, Schwartz LH, Sargent D, Ford R, Dancey J, Arbuck S, Gwyther S, Mooney M, et al: New response evaluation criteria in solid tumours: Revised RECIST guideline (version 1.1). Eur J Cancer 45: 228-247, 2009.

8. Park YH, Lee JL, Ryoo BY, Ryu MH, Yang SH, Kim BS, Shin DB, Chang HM, Kim TW, Yuh YJ and Kang YK: Capecitabine in combination with Oxaliplatin (XELOX) as a first-line therapy for advanced gastric cancer. Cancer Chemother Pharmacol 61: 623-629, 2008.

9. Chu MP, Hecht JR, Slamon D, Wainberg ZA, Bang YJ, Hoff PM, Sobrero A, Qin S, Afenjar K, Houe V, et al: Association of proton pump inhibitors and capecitabine efficacy in advanced gastroesophageal cancer: Secondary analysis of the TRIO-013/LOGiC randomized clinical trial. JAMA Oncol, 2016. 